\title{
GPZL Working Group 1 assesses challenges and a path forward for leprosy during COVID-19
}

\author{
Arielle Cavaliero ${ }^{\mathrm{a}} \&$ Benedict Quao $^{\mathrm{b}}$, On behalf of the Leprosy \\ Emergency Operations Committee, Global Partnership for Zero \\ Leprosy (GPZL) \\ ${ }^{a}$ Leprosy Flagship, Novartis, Switzerland \\ ${ }^{\mathrm{b}}$ National Leprosy Programme, Ghana
}

Submitted and accepted 16 November 2020

\section{Introduction}

The Leprosy Emergency Operations Committee (LEOC) was formed to assess and resolve pandemic-related challenges of national leprosy programmes.

The LEOC aimed to develop a platform for enhancing information sharing, assessing and monitoring the situation in national leprosy programmes, and facilitating the resolution of pandemic-related operational challenges. The LEOC aimed to link experts to address potential needs, assess how to support information needs of national programme managers, and liaise with two other GPZL working groups to exchange information.

\section{Methodology}

The LEOC held 8 meetings from April to August 2020. It facilitated an exchange on the impacts of COVID-19 on MDT supply to compliment the work of WHO and Novartis. Two surveys offered insights from technical experts and experts in the field to better understand distribution during the pandemic.

\section{SURVEY TO NATIONAL LEPROSY PROGRAMME MANAGERS}

The LEOC surveyed national leprosy programme managers to understand their immediate needs and assess their use of a WHO-GPZL-ILEP guidance document on leprosy-related activities during the first months of the pandemic. ${ }^{1}$ The WHO Global Leprosy Programme sent the survey to 222 countries and territories, of which 34 countries, including 10 highpriority countries, responded. The LEOC worked with respondents and countries that did not respond, such as India, to resolve operational challenges. 
SURVEY TO NGOS AND CSOS

The LEOC created a second survey, which reached ILEP members working in endemic countries with a request to forward it to other Civil Society Organizations (CSOs) in countries. Twenty-six organizations from 12 countries responded to the NGO/CSO survey. LEOC members identified distribution issues in the survey responses and followed up with NGOs/CSOs.

\section{Results}

SURVEY TO NATIONAL LEPROSY PROGRAMME MANAGERS

Seventy-six percent of respondents (26 countries) said the outbreak was impacting their programme.

(1) Supply of drugs

Twenty programmes reported that MDT supply had not been affected. However, respondents from 13 countries reported delays in in-country supply, distribution, and/or shortages in facilities. Seven countries reported that although the drugs were available at the health facility level, travel restrictions had hampered patient access. These countries were supplying patients with more than a one-month supply of MDT as much as possible. One programme reported that the MDT supply in country was severely impacted. Others noted issues with the availability of drugs for reactions and challenges in providing continuity of care for ulcers and disabilities.

(2) Supportive services (wound, disability and social care)

One country reported that delays in wound and disability care due to COVID-19 resulted in a large backlog of leprosy patients. Access to treatment was negatively affected by travel restrictions in another country. In a third country, closed clinics restricted MDT supply. Some countries noted issues related to monitoring leprosy reactions, continuity of disability care at all levels, starting community activities, and monitoring of nerve function impairments. One respondent noted that there is a general need for health system strengthening, while another noted the serious impact of COVID-19 on access to basic needs.

(3) General support for the work of national leprosy programmes

Several respondents requested additional guidance, including how to maintain disability services, disability care and self-care groups.

LEOC members discussed survey results, identified the countries most affected, and followed up with programme managers to address needs.

\section{SURVEY OF NGOS AND CSOS}

Common themes included the discontinuation of outreach and community activities, a reduction or elimination of active case finding or screening, and sharp reductions in nerve function tests, disability services and ulcer care. Respondents noted that the MDT supply was usually available, though varied in areas, but patient access was limited. They expressed uncertainty over who was affected by COVID-19 in the leprosy community. Because of lockdowns, travel restrictions and programme closures, NGOs and CSOs reported involvement in humanitarian aid. 


\section{Discussion}

(1) LEOC members facilitated an exchange between technical experts and experts on the ground (NGOs) to expand reach to local partners and collectively analyse supply issues. Tracking and maintaining distribution was the biggest problem, mainly due to travel restrictions. Ten countries required additional follow-up for MDT supply issues. Novartis' Kalwe site associates lived on-site during the lockdown to maintain MDT supply. Novartis supply chain experts and WHO procurement experts then met with incountry representatives and resolved these issues. The LEOC ensured that MDT reached individuals diagnosed with leprosy in a timely manner. Relationships are in place to continuously assess and address future issues.

(2) Leprosy NGOs and CSOs broadened their mandate to address the larger needs of poverty during a time of crisis. ${ }^{4}$ NGOs in India, Bolivia, Nigeria, Bangladesh, Nepal, Myanmar, and Sri Lanka responded to emerging basic needs, such as food and drinking water, and advocated that persons affected by leprosy were not excluded from government relief programmes.

(3) Travel bans have adversely impacted diagnosis and treatment, self-care groups, disability care, ulcer care and the production and fitting of disability appliances/devices. Such bans also hamper active case finding, contact tracing and preventive chemotherapy.

(4) More emphasis on mental health and well-being is needed as COVID-19 has exacerbated the vulnerability of the leprosy community. Pandemics also cause stress and disengagement for health care workers, perhaps requiring different approaches than for patients. ${ }^{3}$

(5) To best support countries in COVID-19 response, GPZL and its members should not only offer guidance. National leprosy programme managers may need additional support to reach measurable outcomes at the country level. Some countries may need technical assistance to implement guidance.

(6) Guidelines should be accessible and widely disseminated. The leprosy community could partner with organizations, such as dermatology associations, so that guidance documents reach more people.

(7) A long-term plan with flexible guidance that accounts for different locations and contexts is needed. Situations have changed since this survey was conducted in April and will continue to change.

\section{Next steps}

The LEOC developed a platform to enhance information sharing, assessing and monitoring the situation in national leprosy programmes. It resolved supply chain issues in many countries. GPZL has established another COVID-19 working group to apply LEOC's learnings alongside programme managers during the pandemic and beyond.

\section{Acknowledgements}

The authors wish to thank all those who responded to the surveys, including national programme managers and non-governmental and civil society organizations. Sincere appreciation to the GPZL Secretariat, Courtenay Dusenbury, Christine Fenenga, and Jessica Cook, for their leadership and dedication, as well as to all the members of the LEOC. 


\section{References}

1 WHO-ILEP-GPZL. Advice about leprosy and COVID-19. Lepr Rev, 2020; 91(2): 228-230. https://leprosyrevie w.org/article/91/2/20-0040.

2 Global leprosy update, 2018: moving towards a leprosy-free world. Weekly Epidemiol Rec, 2019; 94(35/36): 389-412. https://apps.who.int/iris/bitstream/handle/10665/326775/WER9435-36-en-fr.pdf?ua=1.

3 Leszcz M, Maunder R et al. Psychological support for health care workers during the Covid-19 pandemic. CMAJ, 2020; 192: E660. doi:10.1503/cmaj.75864. https://www.cmaj.ca/content/192/24/E660.

4 Molyneux DH, Aboe A, Isiyaku S, Bush S. COVID-19 and neglected tropical diseases in Africa: impacts, interactions, consequences. Int Health, 2020; 12(5): 367-372, https://academic.oup.com/inthealth/article/12/5/ $367 / 5877805$. 